\title{
Saturation numbers for families of Ramsey-minimal graphs
}

\author{
Guantao Chen, Michael Ferrara, Ronald J. Gould, \\ Colton Magnant and John SchmitT*
}

Given a family of graphs $\mathcal{F}$, a graph $G$ is $\mathcal{F}$-saturated if no element of $\mathcal{F}$ is a subgraph of $G$, but for any edge $e$ in $\bar{G}$, some element of $\mathcal{F}$ is a subgraph of $G+e$. Let $\operatorname{sat}(n, \mathcal{F})$ denote the minimum number of edges in an $\mathcal{F}$-saturated graph of order $n$.

For graphs $G, H_{1}, \ldots, H_{k}$, we write that $G \rightarrow\left(H_{1}, \ldots, H_{k}\right)$ if every $k$-coloring of $E(G)$ contains a monochromatic copy of $H_{i}$ in color $i$ for some $i$. A graph $G$ is $\left(H_{1}, \ldots, H_{k}\right)$-Ramsey-minimal if $G \rightarrow\left(H_{1}, \ldots, H_{k}\right)$ but for any $e \in G,(G-e) \not \rightarrow\left(H_{1}, \ldots, H_{k}\right)$. Let $\mathcal{R}_{\min }\left(H_{1}, \ldots, H_{k}\right)$ denote the family of $\left(H_{1}, \ldots, H_{k}\right)$-Ramseyminimal graphs.

In 1987, Hanson and Toft conjectured that

$$
\begin{aligned}
\operatorname{sat} & \left(n, \mathcal{R}_{\min }\left(K_{k_{1}}, \ldots, K_{k_{t}}\right)\right) \\
& = \begin{cases}\left(\begin{array}{c}
n \\
2
\end{array}\right) & n<r \\
\left(\begin{array}{c}
r-2 \\
2
\end{array}\right)+(r-2)(n-r+2) & n \geq r,\end{cases}
\end{aligned}
$$

where $r=r\left(k_{1}, k_{2}, \ldots, k_{t}\right)$ is the classical Ramsey number for cliques.

In this paper, we settle the first non-trivial case of Hanson and Toft's conjecture for sufficiently large $n$ by showing that $\operatorname{sat}(n$, $\left.\mathcal{R}_{\min }\left(K_{3}, K_{3}\right)\right)=4 n-10$ for $n \geq 56$. We also undertake a brief investigation of $\operatorname{sat}\left(n, \mathcal{R}_{\min }\left(K_{t}, T_{m}\right)\right)$ where $T_{m}$ is a tree of order $m$.

AMS 2000 SUBJECT ClASSificAtions: 05C35, 05C55.

KEYWORDS AND PHRASES: Ramsey-minimal, saturation number.

\section{Introduction}

In this paper we consider only graphs without loops or multiple edges. We let $V(G)$ and $E(G)$ denote the sets of vertices and edges of $G$, respectively

${ }^{*}$ This material is based on research done while supported by an ROA Supplement to National Science Foundation (NSF) grant DMS-0758057. 
and we will let $e(G)=|E(G)|$. For any vertex $v$ in $G$, let $N(v)$ and $N[v]=$ $N(v) \cup\{v\}$ denote the neighborhood and closed neighborhoods of $v$, respectively. We denote the complement of $G$ by $\bar{G}$. Given any two graphs $G$ and $H$, their join, denoted $G \vee H$, is the graph with $V(G \vee H)=V(G) \cup V(H)$ and $E(G \vee H)=E(G) \cup E(H) \cup\{g h \mid g \in V(G), h \in V(H)\}$. Finally, for a set of vertices $X$ in $G$, let $\langle X\rangle_{G}$ denote the subgraph of $G$ induced by $X$.

Given a family of graphs $\mathcal{F}$, a graph $G$ is $\mathcal{F}$-saturated if no element of $\mathcal{F}$ is a subgraph of $G$, but for any edge $e$ in $\bar{G}$, some element of $\mathcal{F}$ is a subgraph of $G+e$. If $\mathcal{F}=\{H\}$, then we say that $G$ is $H$-saturated. The classical extremal function $e x(n, H)$ is precisely the maximum number of edges in an $H$-saturated graph of order $n$. Erdős, Hajnal and Moon [5] studied $\operatorname{sat}(n, H)$, the minimum number of edges in an $H$-saturated graph, and determined $\operatorname{sat}\left(n, K_{t}\right)$.

Theorem 1. Let $n$ and $t$ be positive integers such that $n \geq t$. Then

$$
\operatorname{sat}\left(n, K_{t}\right)=\left(\begin{array}{c}
t-2 \\
2
\end{array}\right)+(t-2)(n-t+2) .
$$

Furthermore, $K_{t-2} \vee \bar{K}_{n-t+2}$ is the unique $K_{t}$-saturated graph of order $n$ with minimum size.

For graphs $G, H_{1}, \ldots, H_{k}$, we write that $G \rightarrow\left(H_{1}, \ldots, H_{k}\right)$ if every $k$ coloring of $E(G)$ contains a monochromatic copy of $H_{i}$ in color $i$ for some $i$. A graph $G$ is $\left(H_{1}, \ldots, H_{k}\right)$-Ramsey-minimal if $G \rightarrow\left(H_{1}, \ldots, H_{k}\right)$ but for any $e \in G,(G-e) \not \rightarrow\left(H_{1}, \ldots, H_{k}\right)$. Let $\mathcal{R}_{\min }\left(H_{1}, \ldots, H_{k}\right)$ denote the family of $\left(H_{1}, \ldots, H_{k}\right)$-Ramsey-minimal graphs.

In this paper, we consider $\operatorname{sat}\left(n, \mathcal{R}_{\min }\left(H_{1}, \ldots, H_{k}\right)\right)$ for certain choices of the $H_{i}$. It is straightforward to show that any graph $G$ such that $G \rightarrow$ $\left(H_{1}, \ldots, H_{k}\right)$ must contain a Ramsey-minimal subgraph. Therefore, determining the saturation number for $\mathcal{R}_{\min }\left(H_{1}, \ldots, H_{k}\right)$ is equivalent to determining the minimum number of edges in a graph $G$ of order $n$ with the property that $G \nrightarrow \rightarrow\left(H_{1}, \ldots, H_{k}\right)$ but $G+e \rightarrow\left(H_{1}, \ldots, H_{k}\right)$ for any edge $e$ in the complement of $G$. In 1987, Hanson and Toft [8] discussed this notion and made the following conjecture.

Conjecture 1. Let $r=r\left(k_{1}, k_{2}, \ldots, k_{t}\right)$ be the standard Ramsey number for complete graphs. Then

$$
\operatorname{sat}\left(n, \mathcal{R}_{\min }\left(K_{k_{1}}, \ldots, K_{k_{t}}\right)\right)= \begin{cases}\left(\begin{array}{c}
n \\
2
\end{array}\right) & n<r \\
\left(\begin{array}{c}
r-2 \\
2
\end{array}\right)+(r-2)(n-r+2) & n \geq r .\end{cases}
$$


The statement of this conjecture can also be found in [9].

For $n \geq r$, the fact that $\operatorname{sat}\left(n, \mathcal{R}_{\min }\left(K_{k_{1}}, \ldots, K_{k_{t}}\right)\right) \leq\left(\begin{array}{c}r-2 \\ 2\end{array}\right)+(r-2)(n-$ $r+2)$ arises from consideration of the graph $G=K_{r-2} \vee \bar{K}_{n-r+2}$ which, by Theorem 1 is the unique $K_{r}$-saturated graph of minimum size. Consequently, for any $e \in \bar{G}, G+e$ contains $K_{r}$ and thus $G+e \rightarrow\left(K_{k_{1}}, \ldots, K_{k_{t}}\right)$.

To see that $G \nrightarrow \rightarrow\left(K_{k_{1}}, \ldots, K_{k_{t}}\right)$, consider the coloring obtained by cloning a vertex in any edge-coloring of $K_{r-1}$ containing no $K_{k_{i}}$ in color $i$. For an example when $k_{1}=k_{2}=3$ see Figure 1.

The main result of this paper is that $\operatorname{sat}\left(n, \mathcal{R}_{\min }\left(K_{3}, K_{3}\right)\right)=4 n-$ 10 for $n \geq 56$, settling the smallest non-trivial case of Conjecture 1 for sufficiently large $n$. We also undertake an investigation of the parameter $\operatorname{sat}\left(n, \mathcal{R}_{\min }\left(K_{t}, T_{m}\right)\right)$ where $T_{m}$ is a tree of order $m$.

\section{Main result}

We now proceed by giving our main result.

Theorem 2. For $n \geq 56$,

$$
\operatorname{sat}\left(n, \mathcal{R}_{\min }\left(K_{3}, K_{3}\right)\right)=4 n-10 .
$$

Proof. The fact that $\operatorname{sat}\left(n, \mathcal{R}_{\min }\left(K_{3}, K_{3}\right)\right) \leq 4 n-10$ follows from the coloring of $K_{4} \vee \bar{K}_{n-4}$ described above and pictured in Figure 1.

We therefore aim to prove that $\operatorname{sat}\left(n, \mathcal{R}_{\min }\left(K_{3}, K_{3}\right)\right) \geq 4 n-10$.

Let $G$ be a $\mathcal{R}_{\min }\left(K_{3}, K_{3}\right)$-saturated graph of order $n$ and suppose that $e(G)<4 n-10$. Fix a coloring $\chi$ of $E(G)$ that contains neither a red nor blue $K_{3}$. Throughout the proof, we will attempt to add red or blue edges to $G$. We demonstrate that by modifying $\chi$ (much as Galluccio et al. [7] did in their paper), we may add an edge without creating a monochromatic $K_{3}$, violating our assumption that $G$ is $\mathcal{R}_{\min }\left(K_{3}, K_{3}\right)$-saturated.

It is convenient to visualize $G$ with respect to the coloring $\chi$, and as such we will frequently refer to the "blue graph" and "red graph", as well as the "blue" or "red" degree of a vertex. More formally, let $G_{\text {red }}$ and $G_{\text {blue }}$ denote the subgraphs of $G$ consisting of the edges colored red and blue by $\chi$, respectively. Similarly, for a vertex $v$ in $G, d_{r}(v)$ will denote the number of red edges incident to $v$ and $N_{\text {red }}(v)$ denotes the "red neighborhood" of $v$, that is, the set of vertices $u$ such that $u v$ is red. Similarly, let $N_{r e d}[v]$ denote $N_{\text {red }}(v) \cup v$. We define $d_{b}(v), N_{b l u e}(v)$ and $N_{b l u e}[v]$ in an analogous manner with respect to the blue graph $G_{\text {blue }}$. We will refer to a vertex $x$ in $N_{\text {red }}(v)$ as a red neighbor of $v$ and will also say that the vertices $v$ and $x$ are red-adjacent. 

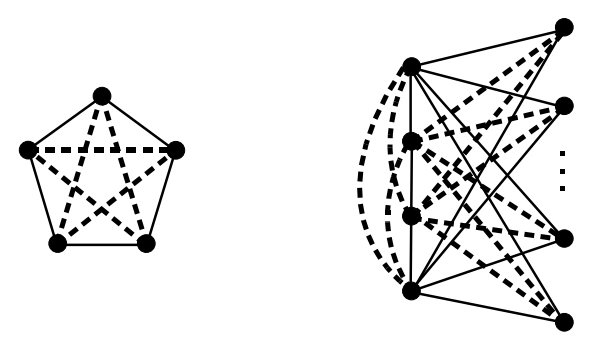

Figure 1: A coloring of $K_{5}$ with no monochromatic triangles that gives rise to a $\mathcal{R}_{\min }\left(K_{3}, K_{3}\right)$-saturated graph.

We now explore the structure of $G=G_{\text {red }} \cup G_{\text {blue }}$, and work toward a contradiction.

Claim 1. The graphs $G_{\text {red }}$ and $G_{\text {blue }}$ are connected.

Proof. Without loss of generality, suppose that $G_{r e d}$ is disconnected, and let $A$ be a component of $G_{r e d}$ having minimum order. Note that for every pair of vertices $a \in A$ and $b \in B=G_{\text {red }}-A$, the edge $a b$ must be in $G_{b l u e}$, since otherwise we could add a missing edge in red without creating a red $K_{3}$. The fact that all of these edges are blue also yields that there are no blue edges within $\langle A\rangle_{G}$ and $\langle B\rangle_{G}$ and hence that both of these (red) subgraphs are $K_{3}$-saturated. Utilizing Theorem 1 , it follows that

$$
e(G) \geq|A||B|+(|A|+|B|-2) .
$$

Since $e(G)<4 n-10$ and $n \geq 56$, we conclude that $|A| \leq 3$.

Since every edge within $B$ is red and $|B| \geq n-3$, there is some edge $u v$ not in $B$. We will add this edge $u v$, colored blue, to $G$ and then modify $\chi$ to remove any blue triangles. Suppose $|A|=3$, and note that since $\langle A\rangle_{G}$ is $K_{3}$-saturated, $A \cong P_{3}$. If we label the vertices of this $P_{3}$, in order, with $a_{1}, a_{2}$ and $a_{3}$, then after adding $u v$ in blue, we recolor $u a_{1}, v a_{2}$ and $u a_{3}$ red. This does not create a monochromatic triangle, and hence contradicts the assumption that $G$ is $\mathcal{R}_{\min }\left(K_{3}, K_{3}\right)$-saturated. The cases where $|A|<3$ are handled in a similar manner.

Since $G$ is $\mathcal{R}_{\min }\left(K_{3}, K_{3}\right)$-saturated, the addition of an edge colored red or blue must create a triangle of that color. The following fact reflects this observation.

Fact 1. If $u$ and $v$ are nonadjacent vertices in $G$, then $N_{\text {red }}(u) \cap N_{\text {red }}(v)$ and $N_{\text {blue }}(u) \cap N_{\text {blue }}(v)$ are both non-empty. 
Also note that if $\alpha(G) \geq n-4$ then $G$ is a subgraph of $K_{4} \vee \bar{K}_{n-4}$. Thus any edge in $E\left(K_{4} \vee \bar{K}_{n-4}\right)$ that is not contained in $E(G)$ could be added to $G$ without destroying the coloring depicted in Figure 1, contradicting the assumption that $e(G)<4 n-10$. This implies the $\alpha(G)<n-4$.

For the remainder of the proof, we let $v$ denote a vertex of minimum degree in $G$ and we let $H$ denote $G-N[v]$. Fact 1 and the assumption that $e(G)<4 n-10$ imply that $2 \leq d(v) \leq 7$.

Galluccio, Simonovits, and Simnoyi [7] investigated $\mathcal{R}_{\min }\left(K_{3}, K_{3}\right)$ saturated graphs, though not just those of minimum size. They gave various constructions of such graphs and various structural results. Useful in establishing our next claim is the following theorem.

Theorem 3. [7] If $G$ is a $\mathcal{R}_{\min }\left(K_{3}, K_{3}\right)$-saturated graph $G$, then $\delta(G) \geq 4$.

Claim 2. $d(v) \geq 4$.

Proof. Follows immediately from Theorem 3.

Let $x$ be a vertex in $H$ and let $v^{\prime}$ be a vertex in $N(v) \cap N(x)$ such that the edges $v^{\prime} v$ and $v^{\prime} x$ are different colors. In this situation, the edge $v^{\prime} x$ does not prevent us from inserting the edge $x v$ in either color, so we call such an edge a wasted edge to $N(v)$. Additionally, say that an edge from $x$ to $N(v)$ is useful if it is not wasted.

Claim 3. $d(v) \leq 5$.

Proof. To begin, suppose that $\delta(G)=d(v)=7$. Every vertex in $H$ must have a common red neighbor and a common blue neighbor with $v$. This implies that

$$
\begin{aligned}
e(G) & \geq 7+\frac{1}{2}\left(\sum_{H} d(x)+e(N(v), H)\right) \\
& \geq 7+\frac{1}{2}(7(n-8)+2(n-8)),
\end{aligned}
$$

which is at least $4 n-10$ for $n \geq 38$.

Therefore assume that $\delta(G)=d(v)=6$. If the subgraph induced by $N(v)$ does not contain a red edge, then we can recolor all six edges incident with $v$ red without a red or a blue $K_{3}$, which contradicts Claim 1 . We could similarly recolor and obtain a contradiction if the subgraph induced by $N(v)$ contained no blue edges. Consequently, there must be at least one blue edge and at least one red edge in the subgraph induced by $N(v)$ and hence at least eight edges in the subgraph induced by $N[v]$. 
Consider a vertex $x$ in $H$ such that $|N(v) \cap N(x)|=2$. By Fact $1, x$ and $v$ must have a common red neighbor $r$ and a common blue neighbor $b$. Suppose that the edge $b r$ is not red (meaning that it may not be in $G$ at all).

Recoloring $x b$ red would leave $x$ and $v$ with no blue common neighbor, so there must be a vertex $v_{r}$ in $G$ that is red-adjacent to both $x$ and $b$. Note that since $r b$ is not red, $r \neq v_{r}$, so $v_{r} \in H$. We also cannot recolor $v b$ red, so there must be a vertex $r_{2} \neq r$ in $N_{\text {red }}(v)$ such that $r_{2} b$ is red. Since $x r_{2}$ is not in $G$, we must not be able to add it to $G_{b l u e}$. Hence there must be some vertex $v_{b}$ in $H$ that is blue-adjacent to both $x$ and $r_{2}$. Note that $v_{b} \neq b$, as $r_{2} b$ is red. We can therefore conclude that for every vertex $x$ having exactly two common neighbors with $v$, there are two vertices in $N(x)-N(v)$, each having a wasted edge.

Every vertex in $G$ has degree at least six, and every vertex in $H$ has at least two edges to $N(v)$. Summing the degrees in $G$, we get that

$$
\begin{aligned}
2 e(G) & \geq 16+2(n-7)+6(n-7)+\sum_{H}\left(d_{N(v)}(x)-2\right)+\sum_{H}(d(x)-6) \\
& =8 n-40+\sum_{H}\left(d_{N(v)}(x)-2\right)+\sum_{H}(d(x)-6) .
\end{aligned}
$$

The vertices $v_{r}$ and $v_{b}$ described above each have at least two useful and at least one wasted edge to $N(v)$, hence they each contribute at least one to $\sum_{H}\left(d_{N(v)}(x)-2\right)$. Furthermore, each of these vertices may be used to prevent recoloring with respect to a number of choices of $x$. Since $v_{r}$ and $v_{b}$ must be adjacent to $x$, they may prevent recoloring with respect to at most three choices of $x$ before beginning to contribute to $\sum_{H}(d(x)-6)$. Consequently, if we let $n_{2}$ denote the number of vertices in $H$ with exactly two neighbors in $N(v)$, we get that

$$
\sum_{H}\left(d_{N(v)}(x)-2\right)+\sum_{H}(d(x)-6) \geq \frac{2 n_{2}}{3}
$$

or that

$$
e(G) \geq 4 n-20+\frac{n_{2}}{3}
$$

Since $e(G)<4 n-10$, it follows that $n_{2} \leq 29$.

There are $n-7-n_{2}$ vertices with three or more neighbors in $N(v)$, implying that

$$
2 e(G) \geq 6(n-7)+2(n-7)+\left(n-n_{2}-7\right)+16
$$


which is a contradiction, given that $n_{2} \leq 29$ and $e(G)<4 n-10$.

Claims 2 and 3 together imply that $\delta(G)$ is either four or five. Next, we eliminate the former possibility.

Claim 4. $\delta(G)=5$.

Proof. Assume that $d(v)=4$ and begin by assuming that $N_{b l u e}(v)=\{b\}$ and $N_{\text {red }}(v)=\left\{r_{1}, r_{2}, r_{3}\right\}$. Note that in order to avoid a monochromatic $K_{3}$, some pair of red neighbors of $v$ must be nonadjacent, say $r_{1}$ and $r_{2}$. Also note that, by Fact $1, b$ must be blue-adjacent to every vertex in $H$. We may therefore assume that each $b r_{i}$ is red since the only red neighbors of $b$ lie in $N_{\text {red }}(v)$, which contains no red edges. Now, since $r_{1}$ and $r_{2}$ are nonadjacent, we may recolor the edges $v r_{1}$ and $v r_{2}$ blue. This forces, by Fact $1, r_{3}$ to be red-adjacent to every vertex $x$ in $H$. Consequently, $H$ must be an independent set. Thus $H \cup\{v\}$ is an independent set of order $n-4$, a contradiction.

Hence, we may assume that $N_{\text {red }}(v)=\left\{r_{1}, r_{2}\right\}, N_{\text {blue }}(v)=\left\{b_{1}, b_{2}\right\}$ and that we cannot recolor the edges incident to $v$ such that $G$ contains no monochromatic $K_{3}$ and $v$ is incident to at most one edge of some color. Along these lines, to prevent us from recoloring $v b_{1}$ red, $b_{1}$ must have a red edge to one of the vertices in $N_{\text {red }}(v)$, say $r_{1}$. Then, to prohibit us from coloring $v r_{1}$ blue, $r_{1} b_{2}$ must be in $G_{b l u e}$. Similarly, we conclude that $b_{2} r_{2}$ must be in $G_{r e d}$ and $b_{1} r_{2}$ must be in $G_{b l u e}$. By Fact 1, every vertex of $H$ must have a red neighbor in $N_{\text {red }}(v)$ and a blue neighbor in $N_{b l u e}(v)$. Hence, the addition of the blue edge $r_{1} r_{2}$ and the red edge $b_{1} b_{2}$ cannot create a monochromatic triangle, so these edges must be present in these colors. Together, this means that the subgraph induced by $N[v]$ is a complete graph composed of disjoint monochromatic 5-cycles, $v b_{1} r_{2} r_{1} b_{2} v$ in blue and $v r_{1} b_{1} b_{2} r_{2} v$ in red.

Since $N(v)$ is complete and $e(G)<4 n-10$, there must be a vertex $x$ in $H$ with three or fewer neighbors in $N(v)$. Suppose that $d_{N(v)}(x)=2$, specifically that $x r_{1}$ is red and $x b_{1}$ is blue. Since $r_{1} b_{1}$ is red, we cannot recolor $x b_{1}$ red, so we will attempt to recolor $x r_{1}$ blue instead. To prevent this, there must be a vertex $x_{b}$, necessarily in $H$, such that $r_{1} x_{b}$ and $x_{b} x$ are both in $G_{b l u e}$. Since $x_{b}$ has a red neighbor in $N_{r e d}(v), x_{b} r_{2}$ must be red. Let $x_{1}, x_{2}, \ldots$ enumerate all possible choices for $x_{b}$ in $G$, and sequentially recolor $x_{i} x$ red if possible. As we cannot recolor all such edges without forcing a contradiction, we may assume that $x_{b} x$ cannot be recolored red. This implies the existence of a vertex $y$, also in $H$, such that $y x$ and $y x_{b}$ are both red. Since $x r_{1}$ and $x_{b} r_{2}$ are both red, this prohibits $y$ from having a red neighbor in $N_{\text {red }}(v)$, a contradiction. 
Suppose then that $d_{N(v)}(x)=3$, specifically that $x b_{1}$ is blue and that $x b_{2}$ and $x r_{1}$ are both red (so that $x b_{2}$ is wasted). We cannot recolor $x r_{1}$ blue, and since $r_{1} b_{1}$ is red, there must be a vertex $x_{b}$ in $H$ such that $x_{b} x$ and $x_{b} r_{1}$ are both in $G_{b l u e}$. Therefore, $x_{b} r_{2}$ must be red and, if we enumerate all possible options for $x_{b}$ as above and sequentially recolor, there must be some choice of $x_{b}$ such that $x_{b} x$ cannot be recolored red. Thus there is a $y$ in $H$ such that $y$ is red-adjacent to both $x_{b}$ and $x$ and once again $y$ cannot have a red neighbor in $N_{\text {red }}(v)$.

Finally, we may assume that $x$ has no wasted edges to $N(v)$, so that (without loss of generality) $x r_{1}$ and $x r_{2}$ are red, while $x b_{1}$ is blue. If we could recolor $x r_{1}$ blue, then $x$ would have a wasted edge, reducing to the previous case. Hence there is some vertex $x_{b}$ such that $x_{b} x$ and $x_{b} r_{1}$ are both blue, and since $b_{1} r_{1}$ is red, $x_{b}$ must be in $H$. Now since $x b_{1}$ and $b_{2} r_{1}$ are both blue, $x_{b}$ cannot be blue-adjacent to $b_{1}$ or $b_{2}$, the final contradiction necessary to complete the claim.

Therefore, $d(v)=\delta(G)=5$.

Claim 5. The vertex $v$ is incident to at least two edges of each color.

Proof. Suppose otherwise, and let $N_{\text {red }}(v)=\left\{r_{1}, \ldots, r_{4}\right\}$ and $N_{\text {blue }}(v)=$ $\{b\}$. By Fact 1, $b$ is blue-adjacent to every vertex in $H$. Moreover, as in the previous claim, we may assume that each of the edges $b r_{i}$ is in $G_{r e d}$.

Let $B$ denote the subgraph induced by $N_{\text {red }}(v)$, necessarily a subgraph of $G_{b l u e}$, which must be triangle-free and hence is bipartite.

Case 1. Suppose $\alpha(B) \geq 3$.

Assume, without loss of generality, that $r_{2}, r_{3}$ and $r_{4}$ are independent in $B$. Since we have assumed that $b$ is red-adjacent to each $r_{i}$, we could recolor each of $v r_{2}, v r_{3}$ and $v r_{4}$ blue without creating a blue triangle. This implies that $r_{1}$ is red-adjacent to every vertex in $H$ and furthermore, since $H \subseteq N_{\text {blue }}(b)$, that $H$ is an independent set. Consequently we could add, in red, any missing edge from $r_{2}, r_{3}$ or $r_{4}$ to $H$ without creating a red triangle, so all of these edges must be present in $G$. We conclude that $G$ has at least $5(n-6)$ edges, a contradiction for $n \geq 20$.

Case 2. Suppose $B \subseteq K_{2,2}$.

Specifically, let $\left\{r_{1}, r_{2}\right\}$ and $\left\{r_{3}, r_{4}\right\}$ be independent sets in $B$. We could recolor $v r_{1}$ and $v r_{2}$ blue, so every vertex $x$ in $H$ must have a red edge to one of $r_{3}$ or $r_{4}$. Symmetrically, each $x$ in $H$ must have a red edge to one of $r_{1}$ or $r_{2}$. Choose some $x$ in $H$ and assume that $x$ is red-adjacent to $r_{1}$ and $r_{3}$ and furthermore that $x r_{2}$ is not in $G$. The only way we are prohibited from adding the edge $x r_{2}$ in blue is if $x$ and $r_{2}$ have a common blue neighbor. Since 
$H \subseteq N_{\text {blue }}(b)$, there are no blue edges in $H$, so this common blue neighbor must be in $N(v)$. Specifically $r_{4}$ must be the common blue neighbor of $x$ and $r_{2}$. Consequently, every vertex $x$ in $H$ must have four neighbors in $N(v)$. This implies that

$$
\begin{aligned}
2 e(G) & \geq \sum_{x \in H} d(x)+e(N(v), H) \\
& \geq 5(n-6)+4(n-6)=9 n-54,
\end{aligned}
$$

contradicting our assumption that $e(G)<4 n-10$ when $n \geq 34$.

For the remainder of the proof of Theorem 2, we will assume that $N_{\text {blue }}(v)=\left\{b_{1}, b_{2}\right\}$ and $N_{\text {red }}(v)=\left\{r_{1}, r_{2}, r_{3}\right\}$.

Claim 6. Every vertex $x$ in $H$ is adjacent to at least three vertices in $N(v)$.

Proof. By Fact 1, each vertex in $H$ is red-adjacent and blue-adjacent to at least one vertex in $N(v)$. Suppose that $x$ in $H$ is red-adjacent to $r_{1}$, blueadjacent to $b_{1}$ and has no other neighbor in common with $v$. Suppose first that $b_{1} r_{1}$ is not in $G_{\text {blue }}$. Note that by Fact 1 we cannot recolor $x r_{1}$ blue, so there must be some vertex $x_{b}$ in $H$ that is blue-adjacent to both $x$ and $r_{1}$. Then $x_{b}$ cannot be blue-adjacent to $b_{1}$, implying that $x_{b} b_{2}$ must be in $G_{b l u e}$. However, we cannot recolor $v r_{1}$ blue since $x$ has only one red neighbor in $N(v)$, so $r_{1}$ must be blue adjacent to either $b_{1}$ or $b_{2}$. Since we assumed $r_{1} b_{1}$ was not in $G_{b l u e}$, this implies that $r_{1}$ is blue-adjacent to $b_{2}$, creating a blue triangle with $x_{b}$.

Therefore, we may assume that $r_{1} b_{1}$ is blue. We cannot recolor $x b_{1}$ red, so there must be some vertex $y$ in $H$ that is red-adjacent to both $x$ and $b_{1}$. This implies that $y b_{2}$ is blue. Once again, let $y_{1}, y_{2}, \ldots$ enumerate all possible choices of $y$, and sequentially recolor the edges $x y_{i}$ blue if possible. As we may not recolor all of these edges, we assume that we have chosen $y$ so that $x y$ cannot be recolored blue. This implies the existence of a vertex $y_{b}$ in $H$ such that $y_{b} y$ and $x y_{b}$ are both blue. However, as in the above claims, $y_{b}$ cannot be blue-adjacent to $b_{1}$ or $b_{2}$, a contradiction.

Claim 7. If a vertex $x$ in $H$ has a wasted edge to $N(v)$, then at least one of the following holds

1. $d_{N(v)}(x) \geq 4$,

2. $d(x) \geq 6$ or

3. $d(x)=5$ and $d_{N(v)}(x)=3$. Also, the two vertices in $N_{H}(x)$ are adjacent and at least one $y \in N_{H}(x)$ has degree at least six. 
Proof. Let $x$ be a vertex in $H$ with a wasted edge to $N(v)$ and suppose that conditions (1) and (2) do not hold.

Case 1. Suppose $x$ has a wasted blue edge.

Assume, without loss of generality, that $x r_{1}$ is red and both $x b_{1}$ and $x r_{2}$ are blue. Furthermore, we first assume that $r_{1} b_{1}$ is not in $G_{b l u e}$. We cannot recolor $x r_{1}$ blue, so there must be a vertex $x_{b}$ that is blue-adjacent to both $x$ and $r_{1}$. Suppose that $x_{b}$ is in $H$, and note that if $x_{b} b_{1}$ was blue we would have a blue $K_{3}$, so $x_{b} b_{2}$ must be in $G_{b l u e}$. Since $x$ has exactly three neighbors in $N(v)$, the edge $v r_{1}$ cannot be recolored blue, so $r_{1}$ must be blue-adjacent to some vertex in $N_{b l u e}(v)$. Since $r_{1}$ and $b_{2}$ are both blue-adjacent to $x_{b}$, we conclude that $r_{1}$ must be blue-adjacent to $b_{1}$, contradicting our assumption that $r_{1} b_{1}$ is not in $G_{b l u e}$. Hence, we may suppose that $x_{b}=r_{2}$ meaning that $r_{1} r_{2}$ is blue.

We cannot recolor $v r_{1}$ blue, as $r_{1}$ is the only red neighbor of $x$. Thus $r_{1}$ must have a blue neighbor in $N_{b l u e}(v)$, so by assumption $r_{1} b_{2}$ must be in $G_{b l u e}$. As $b_{1} b_{2}$ is not in $G_{b l u e}$, the addition of $x b_{2}$ to $G$ in blue forces there to exist a vertex $x_{b}$ that is blue-adjacent to both $b_{2}$ and $x$. Note that $x_{b} \neq r_{2}$ since the blue edge $r_{2} b_{2}$ would create a blue triangle $r_{2} b_{2} r_{1}$, so $x_{b} \in H$. Similarly, as $x r_{3}$ is not in $G$ the addition of $x r_{3}$ in red forces the existence of a vertex $y$ that is red-adjacent to both $r_{3}$ and $x$. Again note that $y \neq r_{1}$ since there can be no red edge within $N_{\text {red }}(v)$, so $y \in H$.

By assumption, $x$ has no more neighbors. Therefore, to prohibit the addition of $x b_{2}$ to $G_{r e d}, x$ and $b_{2}$ must have a common red neighbor. Since $r_{1} b_{2}$ was assumed to be blue, this means that $y b_{2}$ is in $G_{r e d}$ and hence that $y b_{1}$ is in $G_{\text {blue }}$.

Now we wish to show that $d(y) \geq 6$ and $d_{N(v)}(y) \geq 3$. The latter holds as we have shown that $y r_{3}, y b_{1}$ and $y b_{2}$ are in $G$. If $y$ is adjacent to both $r_{1}$ and $r_{2}$, then $d(y) \geq 6$, so suppose first that $y$ is adjacent to neither $r_{1}$ nor $r_{2}$. To prevent adding either $y r_{1}$ or $y r_{2}$ to $G_{b l u e}$, there must be vertices $y_{1}$ and $y_{2}$ such that $y_{1}$ is blue-adjacent to both $r_{1}$ and $y$, and $y_{2}$ is blue-adjacent to both $r_{2}$ and $y$. Note that since $r_{1} r_{2}$ is blue, $y_{1} \neq y_{2}$ and also that $y_{2} \neq b_{1}$ as $x$ is blue-adjacent to both $r_{2}$ and $b_{1}$. Then $d(y) \geq 6$ and $d_{N(v)}(y) \geq 3$. Suppose then that $y$ is adjacent to exactly one of $r_{1}$ or $r_{2}$. If either $y r_{1}$ or $y r_{2}$ is blue, then as $y$ has no other neighbors, it is not possible for $y$ and $x_{b}$ to have a common blue neighbor without creating a blue triangle. The only remaining possibility is that $y r_{2}$ is red, since if $y r_{1}$ was red, $x y r_{1}$ would be a red triangle. However, then the only blue neighbor of $y$ is $b_{1}$, and again it is not possible for $y$ and $x_{b}$ to have a common blue neighbor. We may therefore conclude that $d(y) \geq 6$ and $d_{N(v)}(y) \geq 3$. 
We now show that $x_{b} y$ is in $G$, so that (3) holds. Suppose otherwise, so that $x_{b} y$ is not in $G$. Since we cannot add $x_{b} y$ to $G_{b l u e}$, there must be some vertex $w$ that is blue-adjacent to each of $x_{b}$ and $y$. Furthermore, $w$ cannot be in $N(v)$, as then $w$ would be one of $r_{1}, r_{2}$ or $b_{1}$, creating the blue triangles $r_{1} b_{2} x_{b}, r_{2} x_{b} x$ or $b_{1} x_{b} x$, respectively. Thus $w$ is in $H$, implying by Fact 1 that $w$ must be blue-adjacent to either $b_{1}$ or $b_{2}$, which would create blue $K_{3}$ with $y$ or $x_{b}$, respectively. Thus, the claim holds under the assumption that $r_{1} b_{1}$ is not in $G_{\text {blue }}$.

Suppose that $x r_{1}$ is red and $x b_{1}$ and $x r_{2}$ are blue, but now we also assume that $r_{1} b_{1}$ is in $G_{\text {blue }}$. We cannot recolor $x b_{1}$ red, so there must be a vertex $y$ that is red-adjacent to both $x$ and $b_{1}$. Since $r_{1}$ is the only red neighbor of $x$ in $N(v)$, and $r_{1} b_{1}$ is blue, $y$ must be in $H$. Also, since $y b_{1}$ is red, $y b_{2}$ must be blue.

As in the previous cases, if we enumerate the possible choices of $y$ and sequentially recolor, we may assume that we have selected $y$ such that $x y$ cannot be recolored blue. This implies the existence of a vertex $y^{\prime}$ that is blue-adjacent to both $x$ and $y$. Note that $y^{\prime} \notin H$, since that would imply either $y^{\prime} b_{1}$ or $y^{\prime} b_{2}$ is in $G_{b l u e}$, each of which would lie in a blue $K_{3}$. Therefore $y^{\prime}$ is in $N(v)$, specifically $y^{\prime}=r_{2}$, so that $y r_{2}$ must be blue. Certainly we cannot recolor $x r_{2}$ red, as this would destroy the blue common neighbor of $x$ and $y$ forced above. Hence, there must exist a vertex $y^{\prime \prime}$ with red edges to both $x$ and $r_{2}$. Note that $y^{\prime \prime}$ cannot be $y$ or any vertex in $N(v)$.

The edge $x b_{2}$ cannot be added to $G_{b l u e}$, so there must exist a blue common neighbor of $x$ and $b_{2}$. Since $d(x)<6$ by assumption, this common neighbor must be either $b_{1}$ or $r_{2}$. Either case results in a blue triangle $\left(b_{2} b_{1} v\right.$ or $r_{2} b_{2} y$ respectively), a contradiction.

Case 2. Suppose $x$ has a wasted red edge.

Specifically, assume that $x b_{1}$ and $x r_{1}$ are red and $x b_{2}$ is blue. Again, we first suppose that the edge $b_{2} r_{1}$ is not in $G_{b l u e}$ (so it is either missing or red). We cannot recolor $x r_{1}$ blue so there must exist a vertex $y \in H$ that is blue-adjacent to both $x$ and $r_{1}$. By Fact 1, $y$ must be blue-adjacent to $b_{1}$. Since $v r_{1}$ cannot be recolored blue and we have assumed that $b_{2} r_{1}$ is not in $G_{b l u e}$, there must exist a blue edge from $b_{1}$ to $r_{1}$. This edge forms a blue triangle with $y$, a contradiction.

Hence, we may assume $b_{2} r_{1}$ is blue. The edge $b_{2} x$ cannot be recolored red, so there must exist a vertex $y_{r}$ that is red-adjacent to both $x$ and $b_{2}$. Suppose first that $y_{r} \in H$. By Fact $1, y_{r} b_{1}$ must be blue. If we again let $y_{1}, y_{2}, \ldots$ enumerate the possible choices for $y_{r}$ and sequentially recolor the edges $x y_{r}$ blue, then the fact we cannot recolor all such edges again allows 
us to select $y_{r}$ so that $x y_{r}$ cannot be recolored blue. However, this implies that there is some vertex in $H$ that is blue-adjacent to both $x$ and $y_{r}$. Such a vertex cannot be blue-adjacent to either of $b_{1}$ or $b_{2}$, contradicting Fact 1 and completing the claim.

If, instead, $y_{r}$ is not in $H$, then $y_{r}=b_{1}$. We cannot recolor $v b_{2}$ red, so $b_{2}$ must be red-adjacent to some red neighbor of $v$, say $r_{2}$. Also, adding $r_{2} x$ to $G$ in blue must create a blue $K_{3}$, so there must be a vertex $y^{\prime} \in H$ that is blue-adjacent to both $r_{2}$ and $x$. To avoid creating a blue triangle, $y^{\prime} b_{1}$ must be blue.

Now, for some choice of $y^{\prime}, y^{\prime} r_{2}$ cannot be recolored red so these two vertices have a red common neighbor (possibly $b_{2}$ ). Similarly, $y^{\prime} x$ cannot be recolored red so $y^{\prime}$ and $x$ must have a common red neighbor (possibly $r_{1}$ ). Since $r_{2} x$ is not in $G$ and the addition of $r_{2} x$ in red must therefore create a red triangle, there must be another vertex $y^{\prime \prime}$ that is red-adjacent to both $r_{2}$ and $x$. Since $y^{\prime \prime}$ cannot be $r_{1}$ or $b_{1}$ without creating a red $K_{3}, y^{\prime \prime} \in H$.

Since $d(x)=5$, this must account for all of the neighbors of $x$. We recolor $r_{2} v$ blue since $r_{2}$ has no blue edges to $b_{1}$ or $b_{2}$. However, we still cannot recolor $v b_{2}$ red, so $b_{2} r_{3}$ must be in $G_{r e d}$. We have recolored $v r_{2}$ blue, but we cannot recolor both $v r_{2}$ and $v r_{3}$ blue, so we must have either $r_{2} r_{3}$ or $r_{3} b_{1}$ in $G_{b l u e}$. Finally, as $r_{3} x$ is not in $E(G), r_{3}$ and $x$ must have a common blue neighbor. Note however that $x$ 's blue neighbors are $b_{2}$, which is red adjacent to $r_{3}$ and $y^{\prime}$ which if blue-adjacent to $r_{3}$ would form a blue triangle.

We reach a contradiction, and complete the proof of Theorem 2 by carefully counting the edges of $G$. Partition $H$ into the following sets

- $N_{4}=\{x \in H:|N(x) \cap N(v)| \geq 4\}$

- $N_{3}=\{x \in H:|N(x) \cap N(v)|=3$ and $d(x)=5\}$

- $N_{3}^{*}=\{x \in H:|N(x) \cap N(v)|=3$ and $d(x) \geq 6\}$.

We require one final claim prior to our final count.

Claim 8. If $x \in H$ has no wasted edges, then either $x \in N_{3}^{*} \cup N_{4}$ or $N_{H}(x) \cap\left(N_{3}^{*} \cup N_{4}\right) \neq \emptyset$.

Proof. We may assume $x \in N_{3}$ as otherwise we are done. Suppose first that $x$ has no wasted edges and is blue-adjacent to $b_{1}$ and $b_{2}$ in $N_{b l u e}(v)$ and red-adjacent to $r_{1}$ in $N_{\text {red }}(v)$. We cannot recolor $v r_{1}$ blue, so $r_{1}$ must be blue adjacent to one of $b_{1}$ or $b_{2}$, say $b_{2}$. We cannot recolor $x b_{2}$ red, so $b_{2}$ and $x$ must have a common red neighbor $y_{r}$. Since $r_{1} b_{2}$ is blue, this neighbor must be in $H$ and therefore has a wasted edge to $N(v)$. Then, by Claim 7 , 
either $y_{r} \in\left(N_{3}^{*} \cup N_{4}\right)$ or there is a vertex $y_{r}^{\prime} \in N_{H}\left(y_{r}\right)$ that has degree at least six and is adjacent to $x$. This suffices to demonstrate the claim.

Next, assume that $x$ is blue-adjacent to $b_{1}$ in $N_{b l u e}(v)$ and red-adjacent to $r_{1}$ and $r_{2}$ in $N_{\text {red }}(v)$. We cannot recolor $r_{1} x$ blue so if $r_{1} b_{1}$ is not in $G_{b l u e}$ there is some vertex $y$ in $H$ that is blue-adjacent to both $r_{1}$ and $x$. As above, the desired conclusion would then follow from Claim 7, so we assume that $r_{1} b_{1}$ and, symmetrically, $r_{2} b_{1}$ are both in $G_{b l u e}$. We cannot recolor $b_{1} x$ red, so there must exist a vertex $y$ in $H$ that is red-adjacent to both $b_{1}$ and $x$. Once again, Claim 7 yields the desired conclusion.

Since every vertex in $H$ has at least three edges to $N(v)$ and $\delta(G)=5$, we get that

$$
\sum_{v \in G} d(v) \geq 3(n-6)+5(n-6) .
$$

Each vertex in $N_{3}^{*}$ and $N_{4}$ increases this sum by at least one, and we may improve this bound on $\sum d(v)$ as follows.

$$
\sum_{v \in G} d(v) \geq 3(n-6)+5(n-6)+\left|N_{3}^{*} \cup N_{4}\right|+\sum_{y \in N_{3}^{*} \cup N_{4}}(d(x)-6) .
$$

Let $\Theta:=\left|N_{3}^{*} \cup N_{4}\right|+\sum_{y \in N_{3}^{*} \cup N_{4}}(d(x)-6)$. We claim $\Theta \geq|H| / 4$.

If $\left|N_{3}^{*} \cup N_{4}\right| \geq\left|N_{3}\right| / 3$, then $\Theta \geq\left|N_{3}^{*} \cup N_{4}\right| \geq|H| / 4$ since $V(H)=$ $N_{3} \cup N_{3}^{*} \cup N_{4}$. Suppose then, that $\left|N_{3}^{*} \cup N_{4}\right|<\left|N_{3}\right| / 3$. In this case, we have $\left|N_{3}\right| \geq 3|H| / 4$ and $\left|N_{3}^{*} \cup N_{4}\right| \leq|H| / 4$. Since each vertex $x \in N_{3}$ is adjacent to at least one vertex in $N_{3}^{*} \cup N_{4}$, then the number of edges from $N_{3}$ to $N_{3}^{*} \cup N_{4}$ is at least $\left|N_{3}\right|$.

Since each $y \in N_{3}^{*} \cup N_{4}$ has at least 3 neighbors in $N(v)$, we have

$$
\begin{aligned}
\Theta & =\left|N_{3}^{*} \cup N_{4}\right|+\sum_{y \in N_{3}^{*} \cup N_{4}}(d(y)-6) \\
& \geq\left|N_{3}^{*} \cup N_{4}\right|+\left(\left|N_{3}\right|-3\left|N_{3}^{*} \cup N_{4}\right|\right) \\
& \geq\left|N_{3}\right|-2\left|N_{3}^{*} \cup N_{4}\right| \geq \frac{3}{4}|H|-\frac{2}{4}|H|=|H| / 4 .
\end{aligned}
$$

Thus,

$$
\sum_{v \in G} d(v) \geq 3(n-6)+5(n-6)+\frac{|H|}{4} .
$$

We can augment this sum slightly by counting those edges entirely within $N[v]$. We cannot recolor any blue edge incident to $v$ red and, also, we cannot 
recolor any two red edges incident to $v$ blue. Hence there must be at least four edges completely within $N(v)$ and thus 9 edges completely within $N[v]$. Hence

$$
\sum_{v \in G} d(v) \geq 3(n-6)+5(n-6)+\frac{|H|}{4}+18
$$

a contradiction for $n \geq 46$. This completes the proof of Theorem 2 .

\section{3. $\operatorname{sat}\left(n, \mathcal{R}_{\min }\left(K_{t}, T_{m}\right)\right)$}

In this section we determine $\operatorname{sat}\left(n, \mathcal{R}_{\min }\left(K_{3}, P_{3}\right)\right)$ for $n$ at least 11 , as a contrast to Theorem 2. First, we recall a classic result of Chvátal [3], which states that if $T_{m}$ is any tree of order $m$ then $r\left(K_{t}, T_{m}\right)=(t-1)(m-1)+1$. If we let color one be "red" and color two be "blue", then the lower bound arises from consideration of $(t-1) K_{m-1}$ with every edge colored blue and each edge between the blue cliques colored red. It is well-known that this is the unique edge-coloring of $K_{(t-1)(m-1)}$ with no red $K_{t}$ and no blue $T_{m}$.

Examining the sharpness examples for Theorem 2 and Conjecture 1, it seems reasonable that the correct value of $\operatorname{sat}\left(n, \mathcal{R}_{\min }\left(K_{t}, T_{m}\right)\right)$ may arise from overlapping copies of $K_{(t-1)(m-1)}$ and demonstrating an appropriate coloring. In particular, we obtain the following upper bound.

Proposition 1. Let $t, m$ and $n$ be positive integers and let $T$ be a tree of order $m$. Then $\operatorname{sat}\left(n, \mathcal{R}_{\min }\left(K_{t}, T_{m}\right)\right)$ is at most

$$
\begin{aligned}
& n(t-2)(m-1)-(t-2)^{2}(m-1)^{2}+\left(\begin{array}{c}
(t-2)(m-1) \\
2
\end{array}\right) \\
& \quad+\left\lfloor\frac{n}{m-1}\right\rfloor\left(\begin{array}{c}
m-1 \\
2
\end{array}\right)+\left(\begin{array}{l}
r \\
2
\end{array}\right),
\end{aligned}
$$

where $r \equiv n(\bmod m-1)$.

Proof. Let $H_{1}=K_{(m-1)(t-2)}$ and let $H_{2}=\left\lfloor\frac{n}{m-1}\right\rfloor K_{m-1} \cup K_{r}$ and consider $H=H_{1} \vee H_{2}$. Color each edge in $H_{2}$ blue, and partition the vertices of $H_{1}$ into $t-2$ sets of $m-1$ vertices. Color the cliques induced by each of these sets blue and then color the remaining edges in $H_{1} \vee H_{2}$ red. This coloring contains no red $K_{t}$ and no blue tree of order $m$.

We now wish to show that $H$ is $\mathcal{R}_{\min }\left(K_{t}, T_{m}\right)$-saturated by demonstrating that the coloring of $H$ described above is the unique red/blue coloring of $E(H)$ with no red $K_{t}$ and no blue $T_{m}$. Each copy of $K_{m-1}$ in $H_{2}$ is joined to $H_{1}$, forming a copy of $K_{(m-1)(t-1)}$. The uniqueness of Chvátal's coloring 
assures that in any red/blue edge coloring of $H$ that contains no red $K_{t}$ and no blue $T_{m}$, each of these copies must contain a blue $(t-1) K_{m-1}$ with all other edges red. Consequently, the coloring of each $K_{m-1}$ in $H_{2}$ must be identical. But then, since every vertex of $H_{1}$ lies in a blue copy of $K_{m-1}$, none of these vertices can be blue-adjacent to two components of $H_{2}$, as then this coloring of $H$ would contain a blue $K_{m-1}$ with a pendant edge, and hence a blue copy of $T_{m}$. We therefore conclude that no vertex in $H_{1}$ lies in a blue $K_{m-1}$ with any vertex from $H_{2}$. This implies that each copy of $K_{m-1}$ in $H_{2}$ must be colored blue and that every edge from $H_{1}$ to $H_{2}$ must be red.

We claim next that the component of order $r$ in $H_{2}$ must have every edge colored blue. However, since $H_{1}$ contains a red copy of $K_{t-2}$ and every edge from $H_{1}$ to $H_{2}$ is red, a red edge in this $K_{r}$ would form a red $K_{t}$ in this coloring of $H$, a contradiction.

It remains to show that the addition of any edge, red or blue, to this coloring of $H$ results in either a red $K_{t}$ or a blue $T_{m}$ Note that the only edges in $\bar{H}$ connect vertices in $H_{2}$, so assume that $x$ and $y$ are nonadjacent vertices in $H$. If the edge $x y$ is added in blue, then without loss of generality $x$ lies in a blue copy of $K_{m-1}$ that does not contain $y$. This blue complete graph together with the blue edge $x y$ necessarily contains a blue copy of $T_{m}$. That the addition of $x y$ in red necessarily creates a red $K_{t}$ follows from the observation that $H_{1}$ contains a red copy of $K_{t-2}$ in which every vertex is connected to $x$ and $y$ by a red edge.

For instance, if $t=m=3$, Chvátal's coloring is a red $C_{4}$ with a blue matching and in Figure 2 we give a $\mathcal{R}_{\min }\left(K_{3}, P_{3}\right)$-saturated graph arising from this coloring of $K_{4}$.

This graph has $\left\lfloor\frac{5 n}{2}\right\rfloor-4$ edges and seems like a good candidate for a $\mathcal{R}_{\text {min }}\left(K_{3}, P_{3}\right)$-saturated graph of minimum size. In fact, we can do slightly better.

Theorem 4. For $n \geq 11$,

$$
\operatorname{sat}\left(n, \mathcal{R}_{\min }\left(K_{3}, P_{3}\right)\right)=\left\lfloor\frac{5 n}{2}\right\rfloor-5 .
$$

Prior to proving Theorem 4, we require the following result of Barefoot, et al. [1].

Theorem 5. Let $n \geq 5$ be an integer and let $G$ be a $K_{3}$-saturated graph of order $n$. Then either $G$ is a complete bipartite graph or $2 n-5 \leq e(G) \leq$ $\left\lfloor\frac{(n-1)^{2}}{4}\right\rfloor+1$. 

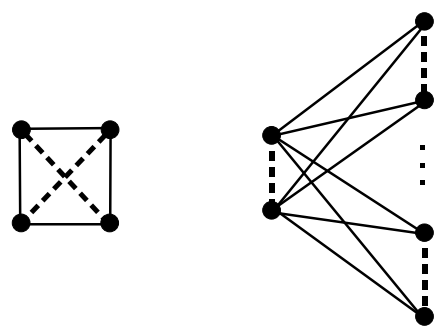

Figure 2: A coloring of $K_{4}$ with no red $K_{3}$ or blue $P_{3}$ that gives rise to a $\mathcal{R}_{\text {min }}\left(K_{3}, P_{3}\right)$-saturated graph.

Let $G$ be a graph and let $v$ be a vertex in $G$. We inflate the vertex $v$ in $G$ by replacing $v$ with an independent set of vertices and connecting each of these new vertices to the neighbors of $v$ in $G$. The next lemma follows directly from the proof of Theorem 5 given in [1], and is also implied by the proof of Corollary 3.1 in [4], so we omit the proof here.

Lemma 1. Let $G$ be a 2-connected $K_{3}$-saturated graph of order $n$ with exactly $2 n-5$ edges. Then $G$ can be obtained by inflating two nonadjacent vertices of $C_{5}$.

We are now ready to prove Theorem 4.

Proof. As above, suppose we are trying to assure either a red $K_{3}$ or a blue $P_{3}$ and let $G$ be a $\mathcal{R}_{\min }\left(K_{3}, P_{3}\right)$-saturated graph. If there exists an edge $e \in G$ which is in three different triangles of $G$, then the only way to edgecolor these triangles without a red $K_{3}$ or a blue $P_{3}$ is to color $e$ blue and the remaining edges red. Hence, we get the following fact which will help us establish our lower bound on $\operatorname{sat}\left(n, \mathcal{R}_{\min }\left(K_{3}, P_{3}\right)\right)$.

Fact 2. In any red/blue edge coloring of a $\mathcal{R}_{\min }\left(K_{3}, P_{3}\right)$-saturated graph $G$ with no red $K_{3}$ or blue $P_{3}$, any edge e lying in three or more triangles must be colored blue.

To establish the upper bound in Theorem 4, consider a copy of $C_{5}$ with vertices $v_{1}, \ldots, v_{5}$ appearing in that order on the cycle. Inflate $v_{1}$ to a set $V_{1}$ of at least three vertices to obtain a graph of order $n \geq 7$ and color all $2 n-5$ edges of this graph red. Next we add a matching, in blue, that consists of the edge $v_{2} v_{5}$ and a maximum matching $M$ amongst the remaining $n-2$ vertices that does not include the edge $v_{3} v_{4}$, as this edge is already present. Call this graph $G_{0}$ and note that the coloring given contains no red $K_{3}$ and no blue $P_{3}$. 
It remains to show that $G_{0}$ is $\mathcal{R}_{\text {min }}\left(K_{3}, P_{3}\right)$-saturated, so consider a red/blue coloring of $E\left(G_{0}\right)$ that contains no red $K_{3}$ and no blue $P_{3}$. Note that the edge $v_{2} v_{5}$ lies in a triangle with each vertex in $V_{1}$, so by Fact 2, it must be colored blue in any coloring with no red $K_{3}$ or blue $P_{3}$. This implies that all of the other edges incident to $v_{2}$ and $v_{5}$ must be colored red. Now we note that each edge in $M-\left\{v_{2} v_{5}\right\}$ is of the form $v_{3} x, v_{4} x$ or $x y$ for vertices $x, y$ in $V_{1}$, which forces each of these edges to be blue, and hence forces the remaining edges in $G_{0}-M$ to be red. Thus we have forced the coloring of $G_{0}$ described above, in which the addition of any edge, in red or blue, forces a red $K_{3}$ or a blue $P_{3}$.

To establish the lower bound, let $G$ be a $\mathcal{R}_{\min }\left(K_{3}, P_{3}\right)$-saturated graph on $n$ vertices with the minimum number of edges. Furthermore, consider a coloring of $G$ containing no red $K_{3}$ and no blue $P_{3}$ having the maximum number of red edges. Notice that if two vertices $u$ and $v$ each have no blue neighbor, then they must be red-adjacent. Hence, the next fact follows immediately.

Fact 3. There is no set $T$ of at least three vertices in $G$ each with no blue neighbors.

As above, let $G_{r}$ and $G_{b}$ denote the graph induced by the red edges and the blue edges in $G$, respectively.

Claim 9. The graph $G_{r}$ is 2-connected.

Proof. Note first that $G_{r}$ is connected, as the addition of a red edge between two components $R_{1}$ and $R_{2}$ in $G_{r}$ could not create a triangle, and hence every edge between $R_{1}$ and $R_{2}$ would have to be in $G_{b}$. This would imply that every edge connecting a vertex in $R_{1}$ and a vertex in $R_{2}$ would be in $G_{b}$, creating a blue $P_{3}$.

Suppose then that $G_{r}$ had connectivity one, and let $v$ be a cut-vertex in $G_{r}$. Let $C_{1}$ and $C_{2}$ be components of $G_{r}-v$ and suppose that there is a vertex $w$ in $C_{1}$ that is not adjacent to $v$. Let $x$ be any vertex in $C_{2}$. Then the edge $w x$ can either be added in red or changed from blue to red, contradicting the choice of $G$ in each case.

Hence, we may suppose $v$ is adjacent to all of $G_{r}-v$ implying that $G_{r}$ must be a star centered at $v$. Examining $G$, there must be a blue matching amongst the vertices of $G-v$. Suppose that $x y$ is an edge of this matching. If we recolor $v x$ blue and $x y$ red, we may then add an edge from $x$ to $G-\{v, x\}$ in red, contradicting the assumption that $G$ is saturated.

The remainder of the proof is broken into cases based on the parity of $n$. 
Case 1. $n$ is odd.

First, we claim that $G_{r}$ is maximal triangle-free. If not, we could either add a red edge to $G$ or recolor a blue edge of $G$ red. Either way, this contradicts our choice of $G$. Since $G_{r}$ is 2 -connected, Theorem 5 yields that $G_{r}$ has at least $2 n-5$ edges. By Fact 3 , there must be at most one vertex (since $n$ is odd) with no incident blue edge. Hence, $e(G) \geq 2 n-5+\left\lfloor\frac{n}{2}\right\rfloor=\left\lfloor\frac{5 n}{2}\right\rfloor-5$, completing this case.

\section{Case 2. $n$ is even.}

The coloring of $G$ was chosen so that the red graph has as many edges as possible, so if there are any edges which could be colored either red or blue without creating a monochromatic $K_{3}$ or $P_{3}$, they will be red. Consequently we may again assume that the red graph is $K_{3}$-saturated but also, by Fact 3 , that there are at most two vertices with no blue neighbor.

If the blue graph is a perfect matching, then by Lemma 1 and the fact that $G_{r e d}$ is 2-connected, $e(G) \geq 2 n-5+\frac{n}{2}=\frac{5 n}{2}-5$, completing the result. Thus, suppose that there is a pair of vertices which are not covered by the blue matching. These vertices must be joined by an edge $e$ in red. We therefore assume, since $G_{r e d}$ is 2 -connected and $K_{3}$-saturated, that $e\left(G_{r e d}\right)=2 n-5$ and furthermore that there are exactly $\frac{n}{2}-1$ blue edges. Note that $e$ can be recolored blue without creating a blue $P_{3}$.

By Lemma 1 , since $n \geq 11, G_{r}$ must be a copy of $C_{5}$ with two nonadjacent vertices inflated. Consider a $C_{5}$ with vertices $v_{1}, v_{2}, \ldots, v_{5}$. Let $v_{1}$ and $v_{3}$ be the inflated vertices (as in the structure provided by Lemma 1) and let $V_{1}$ and $V_{3}$ be the corresponding independent sets. The remainder of the proof is broken into cases based on the location of $e$ in this structure.

If $e=v_{4} v_{5}$, then since $v_{2}$ is adjacent to every vertex in $V_{1}$ and $V_{3}, v_{2}$ has no blue neighbor, a contradiction. Suppose then that $e=v_{4} a_{3}$ for some vertex $a_{3} \in V_{3}$ (or symmetrically $e=v_{5} a_{1}$ for some $a_{1} \in V_{1}$ ). We may then color $e$ blue and add the edge $v_{5} a_{3}$ in red without creating a red triangle. This contradicts the assumption that $G$ was $\mathcal{R}_{\min }\left(K_{3}, P_{3}\right)$-saturated.

Finally suppose that $e=v_{2} a_{3}$ for some $a_{3} \in V_{3}$ (or symmetrically $e=$ $v_{2} a_{1}$ for some $\left.a_{1} \in V_{1}\right)$. Since $G_{b l u e}$ is a matching saturating all of $V(G)-$ $\left\{v_{2}, a_{3}\right\}, v_{5}$ must have a blue neighbor $a_{3}^{\prime}$ in $V_{3}$. Likewise $v_{4}$ must have a blue neighbor $a_{1}^{\prime}$. We may then recolor $v_{5} a_{3}^{\prime}$ and $v_{4} a_{1}^{\prime}$ red, $v_{4} v_{5}$ blue, and add the edge $a_{3}^{\prime} a_{1}^{\prime}$ in blue. This contradicts the assumption that $G$ was $\mathcal{R}_{\text {min }}\left(K_{3}, P_{3}\right)$-saturated and completes the proof. 


\section{Conclusion and open problems}

In this paper, we have verified the first non-trivial case of Conjecture 1. It would be of interest to determine non-trivial lower bounds on $\operatorname{sat}\left(n, \mathcal{R}_{\text {min }}\right.$ $\left.\left(K_{k_{1}}, \ldots, K_{k_{t}}\right)\right)$. For instance, if Conjecture 1 were to hold, a classic result of Spencer [10] would imply that

$$
\operatorname{sat}\left(n, \mathcal{R}_{\min }\left(K_{k}, K_{k}\right)\right) \geq(1+o(1)) \frac{\sqrt{2}}{e} k 2^{\frac{k}{2}} n,
$$

where the $o(1)$ term is with respect to $k$. At this time, we are only able to show the following.

\section{Claim 10.}

$$
\operatorname{sat}\left(n, \mathcal{R}_{\min }\left(K_{k}, K_{k}\right)\right) \geq \frac{(k-1)^{2}-1}{2} n .
$$

Proof. It is readily seen that a graph $H$ satisfies $H \rightarrow\left(K_{k}, K_{k}\right)$ if and only if $H$ contains a $K_{k}$-minimal subgraph $H^{\prime}$. Let $G$ be a $R_{\min }\left(K_{k}, K_{k}\right)$-saturated graph. Then, for any edge $e \in \bar{G}, G+e$ contains a $K_{k}$-minimal subgraph. A result of Burr, Erdős and Lovász [2], reproved recently by Fox and Lin [6], states that the minimum degree of a $K_{k}$-minimal subgraph is at least $(k-1)^{2}$. This implies that the minimum degree of $G$ is at least $(k-1)^{2}-1$, and the result follows.

In addition to Conjecture 1 , one may investigate $\operatorname{sat}\left(n, \mathcal{R}_{\min }(G, H)\right)$ for other pairs of graphs. As a starting point, we conjecture that $\operatorname{sat}\left(n, \mathcal{R}_{\text {min }}\right.$ $\left.\left(K_{t}, T_{m}\right)\right)$ is the same asymptotically as the bound given in Proposition 1.

Finally, as mentioned above, Galluccio, Simonovits and Simonyi have obtained a number of results on (not necessarily minimal) $\mathcal{R}_{\min }\left(K_{3}, K_{3}\right)$ saturated graphs in [7]. The interested reader may wish to investigate the wealth of interesting and challenging conjectures and open problems posed in that paper.

\section{References}

[1] Barefoot, C., Casey, K., Fisher, D., Franaugh, K. and Harary, F. (1995). Size of maximal triangle-free graphs and minimal graphs of diameter 2 . Discrete Math 138 93-99. MR1322083

[2] Burr, S., Erdős, P. and Lovász, L. (1976). On graphs of Ramsey type. Ars Combin. 1 167-190. MR0419285 
[3] Chvátal, V. (1977). Tree-complete graph Ramsey numbers. J. Graph Theory 1 93. MR0465920

[4] Duffus, D. and Hanson, D. (1986). Minimal $k$-saturated and color critical graphs of prescribed minimum degree. J. Graph Theory 10 55-67. MR0830058

[5] Erdős, P., Hajnal, A. and Moon, J. W. (1964). A problem in graph theory. Amer. Math. Monthly 71 1107-1110. MR0170339

[6] Fox, J. and Lin, K. (2007). The minimum degree of Ramsey-minimal graphs. J. Graph Theory 54 167-177. MR2285457

[7] Galluccio, A., Simonovits, M. and Simnoyi, G. (1995). On the structure of co-critical graphs. In: Graph Theory, Combinatorics and Algorithms, Vol. 1, 2 (Kalamazoo, MI, 1992). Wiley-Intersci. Publ., Wiley, New York, 1053-1071. MR1405882

[8] Hanson, D. and Toft, B. (1987). Edge-colored saturated graphs. J. Graph Theory 11 191-196. MR0889351

[9] Pikhurko, O. (2004). Results and open problems on minimum saturated graphs. Ars Combin. 72 111-127. MR2069050

[10] Spencer, J. (1975). Ramsey's theorem - a new lower bound. J. Combin. Theory, Ser. A 18 108-115. MR0366726

Guantao Chen

Georgia State University

Atlanta, GA 30302

USA

E-mail address: gchen@gsu.edu

Michael Ferrara

University of Colorado Denver

DENVER, CO 80217

USA

E-mail address: michael.ferrara@ucdenver.edu

Ronald J. Gould

EMORY UNIVERSITY

Atlanta, GA 30322

USA

E-mail address: rg@mathcs.emory.edu 
Colton Magnant

Georgia Southern University

Statesboro, GA 30460

USA

E-mail address: cmagnant@georgiasouthern.edu

JOHN SCHMiTT

Middlebury College

MidDlEBURY, VT 05753

USA

E-mail address: jschmitt@middlebury.edu

Received OCtober 26, 2010 